\title{
Spiel, Selbst und Gesellschaft \\ Skizze einer Philosophie des Spiels
}

\author{
Núria Sara Miras Boronat
}

Johan Huizinga: Das Spielelement der Kultur. Mit kommentierten Texten von Georges Bataille und Roger Caillois, Berlin 2014. Matthes \& Seitz. 167 S.

Michel Henri Kowalewicz (Hg.): Spiel. Facetten seiner Ideengeschichte, Münster 2013. Mentis Verlag. $253 \mathrm{~S}$.

Alfred Schäfer/Christiane Thompson (Hg.): Spiel, Paderborn 2014. Ferdinand Schöningh. $187 \mathrm{~S}$.

Regine Strätling (Hg.): Spielformen des Selbst. Das Spiel zwischen Subjektivität, Kunst und Alltagspraxis. Bielefeld 2012. Transcript Verlag. 307 S.

Alle Wesen spielen und haben immer gespielt. Menschen aller Lebensalter, Kulturen, Geschlechter, Status und politischer Einstellungen. Aber wir wissen durch unseren Sprachgebrauch, dass auch Götter, Tiere, das Meer und die Erde im metaphorischen Sinne spielen können. Warum dann aber hat eine Philosophie des Spiels so große Schwierigkeiten, sich als seriöse und eigenständige Philosophie zu behaupten? Wir können vorläufig zwei Gründe dafür angeben, die die Anerkennung einer Philosophie des Spiels erschweren. Erstens sind die Phänomene, die wir »Spiele» nennen, derart heterogen und verschiedenartig, dass es noch keinen Konsensus innerhalb der Spielforschung darüber gibt, was als "Spiel« zählen darf. Die Vielfältigkeit der Spiele stellt eine echte Herausforderung für die Philosophie dar, wenn wir die Spielphilosophie als Suche nach einer eindeutigen und endgültigen Definition des Spiels verstehen. Eine weitere Schwierigkeit, wie schon Huizinga in Homo Ludens bemerkt hat ${ }^{1}$, liegt in der Tatsache, dass die verschiedenen Weltsprachen mehrere Wörter benutzen, die das semantische Feld des Ludischen abdecken und dabei oft an den Grenzen des Ludischen stehen. Die semantischen Felder des Ludischen und ihre jeweiligen Assoziationen in den verschiedenen Weltsprachen decken sich nicht unbedingt. Diese Ungleichheit hat Konsequenzen nicht nur für die Definition des Spiels, sondern auch für die Ansprüche einer allgemeingültigen Spielontologie. Deswegen wurde viel häufiger das Spiel durch seinen Gegensatz definiert: Spiel ist kein Ernst, keine Arbeit, nichts Wirkliches, usw ${ }^{2}$. Es fragt sich aber, wie weit wir mit dieser Auflistung

\footnotetext{
${ }^{1}$ Johan Huizinga: Homo ludens: Vom Urprung der Kultur im Spiel, Reinbek bei Hamburg, 2011.

${ }^{2}$ Natascha Adamowsky argumentiert sehr überzeugend, dass diese Strategie, die das Spiel durch seinen Gegensatz zu definieren sucht, das Wesen des Spiels verfehlt: „Die Differenzierung Spiel - Nicht-Spiel führt somit auf die Logik einer Freund-Feind-Unter-
} 
von Gegensätzen in dem Verständnis davon, was ein Spiel ausmacht, kommen. Dieser Ansatz verschafft lediglich Klarheit darüber, was ein Spiel nicht sein kann, sprich: wo Spiele für uns an ihre Grenzen stoßen. Zweitens mag die Philosophie vielen genau als dieses Gegenteil des Spiels erscheinen. Philosophie strebt nach Ordnung, Deutlichkeit, Unterscheidung, Rationalität, während das Spiel unter diejenigen Gegenstände zu fallen scheint, die das Andere der Philosophie oder der Vernunft hervorheben: Unordnung, Ambivalenz, Zweideutigkeit, Irrationalität ${ }^{3}$.

Diese Schwierigkeiten der Philosophie, das Spiel als ihr eigenes Objekt anzuerkennen, lassen sich anhand von zwei paradigmatischen Beispielen sowohl aus der Philosophie als auch aus der Kunst illustrieren. Das erste Beispiel taucht in Ludwig Wittgensteins Philosophische Untersuchungen auf. Wittgenstein erfindet das Wort »Sprachspiel« mit zwei Hauptzielen. Zum einen, um die Mannigfaltigkeit der Sprache durch die Assoziation von Satzarten mit Spielarten zu erhellen. Zum zweiten, um die innere Verbindung von Sprache und Tätigkeit durch das Wort »Sprachspiel« hervorzuheben. Wittgenstein will dem Leser die Mannigfaltigkeit der Sprachspiele vor Augen führen, indem er mehrere Beispiele von Sprachverwendungen in eine Liste einträgt:

»[...] Berichte eines Hergangs -

Über den Hergang Vermutungen anstellen -

Eine Hypothese aufstellen und prüfen -

Darstellen der Ergebnisse eines Experiments durch Tabellen und

Diagramme -

Eine Geschichte erfinden; und lesen -

Theater spielen -

Reigen singen -

Rätsel raten -

Einen Witz machen; erzählen -

scheidung zurück, die den eigentlichen Wesenszug des Spiels ausblendet, eine Bewegung im Zwischen, eine Begegnung zu sein. Seit gut zwei Jahrhunderten wird der kulturkonstitutive Status des Spiels auf eine reine Oppositionsrolle zurückgeschnitten. [...] Hier ist es wichtig, Missverständnisse zu vermeiden. Natürlich unterscheidet sich das Spiel von vielen Dingen. Es ist aber erkenntnistheoretisch nicht zwingend, Eigenarten eines Phänomens als Opposition zu etwas anderen zu beschreiben. Niemand käme auf die Idee, Platon als Nicht-Aristoteles zu beschreiben, Goethe als Nicht-Shakespeare, Willy Brandt als NichtKohl. Im besten Falle sind solche Oppositionen nur banal. Seltsamerweise scheint dies bei Spielen kaum jemandem aufzufallen." (Adamowsky in STRÄTLING-Band, S. 61).

${ }^{3}$ Alexander Aichele hat gegen diese streng rationalistische Auffassung der Philosphie plädiert, indem er das Spielerische im Philosophieren Platons, Kants und Nietzsches erforscht hat. Siehe: Alexader Aichele: Philosophie als Spiel: Platon, Kant, Nietzsche, Berlin, 2000 . 
Ein angewandtes Rechenexempel lösen Aus einer Sprache in die andere übersetzen Bitten, Danken, Fluchen, Grüßen, Beten. $\aleph^{4}$

Selbstverständlich beziehen sich diese Beispiele nicht nur auf Spiele. Sie sind eher Muster von der Flexibilität unserer Sprachen, die eine breite Spanne von Funktionen erfüllen können, von den primitivsten zu den komplexesten. Nach Wittgenstein gehört dies zu unserer natürlichen Ausstattung: »Befehlen, fragen, erzählen, plauschen gehören zu unserer $\mathrm{Na}$ turgeschichte so wie gehen, essen, trinken, spielen. $\ll^{5}$ Der Rekurs auf das Spiel ist keine Antwort auf die Frage, was alle diese Sprachvorgänge gemeinsam haben oder was das Wesen der Sprache sein mag. Er will es auch nicht sein. Wittgenstein verzichtet nicht auf eine Sprachphilosophie, aber er verzichtet auf eine uniforme Bestimmung der Sprache, die deren Plastizität nicht gerecht würde. Stattdessen ist es Wittgensteins Vorschlag, eine monolithische Bestimmung des Sprachwesens durch das vieldeutige Bild von Familienähnlichkeiten zu ersetzen. Das Bild der Familienähnlichkeiten fällt Wittgenstein vielmehr gleichzeitig mit dem Bild von Sprachspielen ein, wo er auf eine alternative Bedeutungstheorie zu der von ihm im Tractatus Logico-Philosophicus aufgeworfenen abzielt ${ }^{6}$. Wir haben dasselbe Problem, wenn wir versuchen, das Wesen der Sprache und das Wesen der Spiele ein für alle Mal zu bestimmen: »die Bestrebung, nach etwas Ausschau zu halten, das all den Dingen gemeinsam ist, die wir gewöhnlich unter einer allgemeinen Bezeichnung zusammenfassen. Wir sind z. B. geneigt zu denken, daß es etwas geben muß, das allen Spielen gemeinsam ist, und daß diese gemeinsame Eigenschaft die Anwendung der allgemeinen Bezeichnung "Spiel« auf die verschiedenen Spiele rechtfertigt; während Spiele doch eine Familie bilden, deren Mitglieder Familienähnlichkeiten haben. Einige haben die gleiche Nase, einige die gleichen Augenbrauen und andere wieder denselben Gang; und diese Ähnlichkeiten greifen ineinander über. ${ }^{7}$ Solche philosophischen Probleme, die mit der Suche eines allgemeinen Begriffs verbunden sind, entstehen, "wenn die Sprache fei$\operatorname{ert}^{8}{ }^{8}$. Wittgenstein hält eine ausgesprochene Definition des Spiels für un-

${ }^{4}$ Ludwig Wittgenstein: Philosophische Untersuchungen, \23, Frankfurt am Main, 1984, S. 250.

${ }^{5}$ Ludwig Wittgenstein: Philosophische Untersuchungen, $\$ 24$, Frankfurt am Main, 1984, S. 251

${ }^{6}$ Stefan Deines betont diesen "Zufall« auch in der Entstehung von Wittgensteins Spätphilosophie (Deines in STRÄTLING-Band, S. 24).

${ }^{7}$ Ludwig Wittgenstein: Das Blaue Buch. Eine philosophische Betrachtung (Das Braune Buch), Frankfurt am Main, 1984, S. 37.

${ }^{8}$ Ludwig Wittgenstein: Philosophische Untersuchungen, $\$ 38$, Frankfurt am Main, 1984, S. 260. 
nötig, immer wenn wir unser Wissen darüber, was Spiele sind und was nicht, durch Analogien und Beschreibungen erklären können ${ }^{9}$. Wie wir keiner klaren Vorstellung von Regeln bedürfen, um sie richtig anwenden zu können, so brauchen wir auch keine feste Beschreibung dessen, was ein Spiel ist, um es zu spielen - und es zu genießen. Wittgenstein hat dank seines Konzepts der Sprachspiele nicht nur das Spielen wieder in die Philosophie eingeführt, sondern durch es gleichzeitig Wege einer Philosophie eröffnet, die mit der Unbestimmtheit der Phänomene umgehen kann, ohne sich selbst in dieser Unbestimmtheit zu verlieren. Zudem haben wir es mit einem Philosophen zu tun, der gerne mit der Sprache spielt, um uns zu zeigen, wann das Streben nach Allgemeinheit ein Hindernis für unsere Untersuchungsmethode sein kann. ${ }^{10}$

Das zweite Beispiel entnehmen wir der Kunst: das Gemälde »Die Kinderspiele« (datiert um 1560) von Pieter Brueghel dem Älteren, das heute im Kunsthistorischen Museum Wien zu sehen ist. Auf dem Gemälde werden über 80 Kinderspiele in den Niederlanden des 16. Jahrhunderts dargestellt. Man erblickt Jungen und Mädchen in Gruppen, die sich isoliert betrachten lassen. Einige Spiele werden noch heute gespielt, so zum Beispiel: Blinde Kuh, Drehen im Kreis, Tauziehen, Kreisel, Puppen oder Hochzeit. Wir können dieses Gemälde als eine Momentaufnahme des Alltagsleben jener Zeit und jenes Ortes betrachten und die Frage aufwerfen: Was sagen diese Spiele über die Kultur und die Zeit, in denen sie gespielt wurden? Ändern sich Spiele mit der Zeit? Wie hängt das Spielen mit anderen kulturellen Praktiken zusammen? Ist das Spiel ein Grundphänomen oder eher eine Marginalie in der Kultur? Wie verhalten sich Menschen im Spiel? Wer ist das Subjekt des Spiels? Diese Fragestellungen ließen sich unter verschiedene Forschungsgebiete subsumieren: Ontologie (Was macht das Spiel aus?), Begriffsgeschichte (Wie wird das Spiele durch verschiedene philosophische Ansätze definiert?), Geschichtsphilosophie (Wie ändert sich das Spielen mit der Zeit? Was sagt das Spiel über seine Zeit aus?) oder Ethik (Wer ist das Subjekt des Spiels? Wo hört das Spielen auf?), und benannt wären damit nur einige mögliche. Diese diversen Fragestellungen sind mit den jungen Kulturwissenschaften und der Soziologie ins Leben gerufen worden.

Nehmen wir drei paradigmatische Fälle von Sozialwissenschaftlern, die sich mit dem Spiele befasst haben: Johan Huizinga, Roger Caillois und

\footnotetext{
${ }^{9}$ Ludwig Wittgenstein: Philosophische Untersuchungen, \75, Frankfurt am Main, 1984, S. 282.

10 "Dieses Streben nach Allgemeinheit ist das Ergebnis einer Anzahl von Bestrebungen, die mit bestimmten philosophischen Verwirrungen verbunden ist." Ludwig WitTGENStein: Das Blaue Buch. Eine philosophische Betrachtung (Das Braune Buch), Frankfurt am Main, 1984, S. 37.
} 
Brian Sutton-Smith. Diese drei Theoretiker teilen fundamentale Intuitionen über das Spiel und die Kultur. Erstens, das Spiel ist ein eigenständiges Phänomen, dessen Definition nicht auf andere Phänomene zurückgeführt werden darf. Das Spiel ist nicht etwas Sekundäres oder Marginales in der Kultur. Umgekehrt ist das Spiel vielmehr ein Totalphänomen ${ }^{11}$, auf das andere kulturelle Phänomene zurückgeführt werden können oder müssten. Zweitens, die Einstellung des Subjekts im Spiel ist ein wichtiges Element dieser Definition. Drittens, die Abwandlung der Spielformen ist repräsentativ für einen bestimmten Zeitgeist und bringt sogar eine Kulturdiagnose zum Ausdruck. Die bis heute kanonische Definition des Spiels verdanken wir Johan Huizinga: "Spiel ist eine freiwillige Handlung oder Beschäftigung, die innerhalb gewisser festgelegter Grenzen von Zeit und Raum nach freiwillig angenommenen, aber unbedingt bindenden Regeln verrichtet wird, ihr Ziel in sich selber hat und begleitet wird von einem Gefühl der Spannung und Freude und einem Bewußtsein des `Andersseins als das gewöhnliche Leben «"2. Roger Caillois fand Huizingas Definition unzureichend und fügte ihr eine Klassifizierung hinzu, die größere Spielefamilien mit lateinischen Namen erfasst: Agon (Kampf), Alea (Glück), Mimicry (Nachahmung) und Ilinx (Rausch). Sutton-Smith wiederum stellt hunderte von Spielen und spielähnlichen Tätigkeiten in einer Liste von acht Spielkategorien zusammen ${ }^{13}$, ohne dabei in seinen Augen jedoch ihrer zeitlichen und räumlichen Vielfältigkeit Rechenschaft zu tragen. Seine Lösung der Schwierigkeit hingegen ist originell und unserer Zeit gemäß: Er nennt sie »die rhetorische Lösung«. Spiele werden in sieben verschiedenen Rhetoriken unterteilt, die folgende Elemente kombinieren: Epoche, Funktion, bevorzugte Spielform, Spieler, Disziplin und Spieltheoretiker ${ }^{14}$. Die sieben Rhetoriken, die auch sieben Spielnarrative darstellen, bilden zwei Gruppen: antike Rhetorik (Schicksal, Macht, Identität, Frivolität) und die moderne Rhetorik (Fortschritt, Selbst, das Imaginäre) ${ }^{15}$. Aus dieser multidimensionalen Kombination ergibt sich zum Beispiel die Charakterisierung der Rhetorik von Spiel und Imaginärem wie folgt: Romantik (Epoche), Kreativität und Flexibilität (Funktion), Phantasie (Spielform), Schauspieler (Spieler), Kunst und Literatur (Disziplin), Schiller, Bachtin,

11 Roger Caillois: Die Spiele und die Menschen: Maske und Rausch, Ullstein, 1992.

${ }^{12}$ Johan Huizinga: Homo Ludens, S. 37.

13 Brian Sutton-Smith: The Ambiguity of Play, Cambridge/London, 2001, S. 4-5.

14 Brian Sutton-Smith: The Ambiguity of Play, Cambridge/London, 2001, S. 15-17; 215.

15 »The rhetoric of progress, the rhetoric of the self, and the rethoric of the imaginary constitute the modern set of rhetorics, with a history largely elaborated ideologically only in the past two hundred years." Brian Sutton-Smith: The Ambiguity of Play, Cambridge/ London, 2001, S. 11. 
Derrida und Bateson (Spieltheoriker) ${ }^{16}$. Was die Einstellung des Subjekts innerhalb des Spiels angeht, ist für alle drei Spieltheoretiker sein Auftreten im Spiel als eine separate Domäne charakteristisch, auch wenn sie diese Domäne anders konzipieren: Huizinga fokussiert das Anderssein als normales Leben; Caillois belebt die ehemalige Bedeutung des jeu als persönlichen Stil, der sich im Spiel manifestiert; Sutton-Smith sieht das Spiel als wichtigen Teil unserer Anpassung an die Welt, denn zu spielen helfe allen Kreaturen mit Kontingenz umzugehen. Von Sutton-Smiths Standpunkt aus ist das Spielen ein Modell für unseren alltäglichen Existentialismus ${ }^{17}$. Und schließlich haben unsere Autoren die Erforschung des Spiels zu einer epochalen Diagnose vereint. Schon Im Schatten von Morgen aus dem Jahre 1935 hat Huizinga auf den Puerilismus unserer Zeit hingewiesen: Wir sind nicht mehr in der Lage, Spiel von Nicht-Spiel zu unterscheiden, weil wir die Grenzen bereits verschwommen lassen haben. Platon hatte die Menschen noch die Spielzeuge der Götter genannt; heute gebrauchen die Menschen ihre Welt selbst wie ein Spielzeug ${ }^{18}$. Caillois, der Huizingas geschichtsphilosophische These scharf kritisiert, behauptet sogar, dass die Präferenz für eine Spielfamilie das Schicksal einer Zivilisation entscheiden könne. Wie es demnach aussieht, hat sich unsere postmoderne Welt für Rausch und Mimicry entschieden. Sutton-Smith hat die epochale und serielle Bedeutung des Spiels mit seiner rhetorischen Lösung herausgestellt. Genau dieses wiederbelebte Interesse am Spiel aber zeichnet unsere Zeit aus: Wir leben in der Zeit des Ludic Turns ${ }^{19}$.

Wenn wir heute eine Momentaufnahme, wie diejenige von Peter Brueghel dem Älteren, von unseren Spielen machen würden, würden wir auch sicherlich erstaunt sein, über die auffälligen Unterschiede zwischen Bruegehls Spielen und unseren. Zuerst würden wir wahrscheinlich nicht mehr ein malerisches Medium vor uns haben. Stattdessen würden wir wahrscheinlich eine Collage mit vielen Handy-Photos komponieren. Das Handy selbst ist zu einem Spielzeug für Kinder und Erwachsene geworden. Ein weiterer Unterschied wäre vielleicht, dass im Vergleich zu Brueghels Gemälde, die meisten Menschen nicht mehr auf der Straße spielend zu sehen wären. Hauptsächlich spielen wir nicht mehr gemeinsam auf dem Spielplatz: Spielplätze und Spielpartner begleiten uns überall hin, ohne überhaupt noch körperlich fassbar sein zu müssen. Wir würden wahrscheinlich Menschen sehen, die zuhause am Computer sitzen oder mit dem Handy im Bus herumtollen. Die digitalen Medien haben unsere

\footnotetext{
16 Brian Sutton-Smith: The Ambiguity of Play, Cambridge/London, 2001, S. 127-150; 215.

17 Brian Sutton-Smith: The Ambiguity of Play, Cambridge/London, 2001, S. 228.

${ }^{18}$ Johan Huizinga: Kultur - und zeitkritische Schriften, Paderborn, 2014, S. 93-99.

19 Brian Sutton-Smith: The Ambiguity of Play, Cambridge/London, 2001, S. IX.
} 
gesellschaftlichen Kontexte revolutioniert - und damit auch die Spielformen sowie die Spieler selbst. Dementsprechend zeitigen diese Transformationen auch Konsequenzen für die Spieltheorie. Heute besteht eine Erforschung von Games als geschlossene Systeme mit inhärenten Ziele neben der traditionellen Auffassung vom Free Play als Ausdruck persönlicher Kreativität. So haben beispielsweise die Game-Designer Katie Salen und Eric Zimmerman 2004 definiert, was unter einem game zu verstehen ist: "A game is a system in which players engage in an artificial conflict, defined by rules, that results in a quantifiable outcome. ${ }^{20}$. Die Entscheidung zwischen Game oder Play ist dabei keine triviale: Game und Play scheinen einander auszuschließen. Sich auf Games zu konzentrieren, bedeutet nur diejenigen Spieltätigkeiten in Betracht zu ziehen, bei denen Gewinn und Verlust möglich sind, d.h. bei denen Regeln, Entscheidungsprozesse und Strategien sich als wesentliche Züge des Spiels erweisen. Das Play als primäres Phänomen zu betrachten, bedeutet hingegen das Moment der Konkurrenz aus dem Spiel zu lassen und stattdessen Kreativität, Expressivität und Freiheit in den Vordergrund zu rücken. Das Play als freie, möglichst indeterminierte Tätigkeit darf nicht instrumentalisiert werden, um Ziele ausserhalb seiner selbst zu erreichen, wie es etwa beim Sport oder Wettbewerben der Fall ist. Dabei handelt es sich nicht nur um eine philosophische, sondern gleichermaßen um eine ideologische Entscheidung.

Die Spielforschung ist zur Zeit höchst lebendig und stimulierend. Sie eröffnet interessante Wege für die Erläuterung von Kategorien und Begriffen wie Konflikt, Konkurrenz, Performativität, Kunst und/oder Künstlichkeit, Freiwilligkeit, Ungewissheit, Lern -und Entscheidungsverfahren, Wirklichkeit, Virtualität, Genuss, Kontrollübernahme, Sozialität und andere. Die vier Bände, die in dieser Besprechung eingeführt werden, beinhalten insgesamt 42 Beiträge zum Thema Spiel im Zeitraum von 2012 bis 2014. Die Beiträge versammeln Ansätze von sehr verschiedenen Disziplinen (u.a. Geschichte, Soziologie, Kunstwissenschaft, Literaturwissenschaft, Medienwissenschaft, Pädagogik, Philosophie) und bezeugen gleichzeitig die Pluralität innerhalb dieser Disziplinen. Sie exemplifizieren auch, dass eine sachgemäße Erörterung des Spielproblems zunehmend interdisziplinäre Arbeit erfordert. Doch die hier diskutierten Beiträge bieten letztlich nur ein kleines Muster von dem, was eine zeitgenössische Momentaufnahmen a là Brueghel zu leisten hätte. Zudem bildet dieses Muster keine eigentliche Einheit, so dass sie hier lediglich unter drei der wichtigsten Aspekte diskutiert werden, die aus Sicht der Rezensentin den Ludic Turn ausmachen: Spiel (Was ist das Spiel oder das Spielen?), Selbst (Was ist

${ }^{20}$ Katie Salen/Eric Zimmerman: Rules of Play. Game Design Fundamentals, Cambridge, MA/London, 2004, S. 80. 
das Subjekt des Spiels und im Spiel?) und Gesellschaft (Was sagt das Spiel oder das Spielen über seinen Kontext aus?). Es scheint an der Zeit, dass die Philosophie sich auf das Spiel einlässt.

Die vier Beiträge in dem von Knut Ebeling herausgegebenen Band Das Spielelement in der Kultur beschreiben die ersten Schritte der Kulturwissenschaft, die gleichzeitig der Spielforschung Antrieb verlieht. Ausgehend von der Spiel-Thematik warten die Texte mit mindestens zwei Überlegungen auf, die junge Kulturwissenschaft als neues Fachwissen zu etablieren. Erstens ist da der Anspruch, sich von der Geschichtsphilosophie und der philosophischen Ästhetik abzugrenzen und sich als eigenständige Disziplin zu begründen. Zweitens nutzt man die Möglichkeit, von der gleichaltrigen empirischen und ethnographische Forschung zu profitieren und sich dennoch vor ihr auszuzeichnen, indem man eine allgemeingültigere Charakterisierung der menschlichen Kultur unternimmt. Den Anstoß hierzu bildet Huizingas Homo Ludens (1938), mit dem ein zutiefst originelles und aufschlussreiches Werk vorliegt, das die neue Wissenschaft ins Leben ruft. Huizingas These ist bereits vom Ansatz her höchst provokativ: "Die großen ursprünglichen Betätigungen des menschlichen Zusammenlebens sind alle bereits von Spiel durchwoben ${ }^{21}$. Die Vorentwürfe dieser These in Huizingas Rektoratsrede von 1933 verkünden ein unerhörtes intellektuelles Wagnis. Huizingas Abenteuerlust wird in diesem kurzen Text schon vernehmbar.

$\mathrm{Zu}$ einer eigenständigen Disziplin gehört prinzipiell eine Forschungsgemeinschaft, die sich als solche bekennt und in der ein produktiver Austausch über ihr Themenfeld entsteht. Die Textsammlung des Bandes bekundet entsprechend die unmittelbare Rezeption von Huizingas epochaler Entdeckung des Spiels als Bewertungsobjekt der Kultur in Deutschland und Frankreich. Die Grundzüge der Auseinandersetzung über den Gründungsbereich der Kulturwissenschaft, ausgehend von verschiedenen Ansatzpunkten, lässt sich daraus trefflich rekonstruieren. Dabei verdeutlichen sie gleichwohl die fundamentalen Missverständnisse und Gegenstände dieses zum Teil unerfüllten Dialogs. Interessanterweise wird in der Sammlung Huizingas Rektoratsrede wiedergegeben, bei der der Keim seiner späteren Überlegungen schon vorhanden ist. Huizingas Ansprüche sind hier teilweise kühner und verspielter formuliert, wie Knut Ebeling schön bemerkt (9). Huizinga ergänzt diese noch mit dem rohen philosophischen und empirischen Material, das später in Homo Ludens viel konsequenter

21 Johan Huizinga: Homo Ludens, S. 12. 
verarbeitet wird. Aber die Beiträge von Eric Voegelin, Roger Caillois und Georges Bataille beziehen sich vorrangig auf den Homo Ludens, der eine reifere und systematischere Leistung Huizingas darstellt. Dadurch wird der Eindruck erweckt, dass dieser Austausch viele wichtige Elemente auBer Acht gelassen hat, und dass wir jetzt in einer besseren Position befinden, die Gründe hierfür einzuschätzen. Knut Ebelings Vorwort und Nachwort macht uns mit dem geschichtlichen und theoretischen Zusammenhang vertraut, so dass wir uns besser eine Meinung bilden können und suggeriert dabei zugleich viele andere Wege, die zu beschreiten jetzt die Zeit gekommen scheint. Es wäre vielleicht wünschenswert, dass die Auswahl der Texte ausführlicher für Nicht-Eingeweihte begründet würde. Während die Linie Huizinga-Caillois-Bataille eine Tradition innerhalb der Spielphilosophie bildet, hat man zu Anfang das seltsame Gefühl, dass Vogelins Text außerhalb dieses Dialogs stünde. Er hatte zwar einen persönlichen und philosophischen Bezug zu Huizinga, jedoch nicht zu den anderen Autoren, was dieses Gefühl von einem separaten Gespräch vermitteln mag. Trotzdem ergibt sich aus der gemeinsamen Lektüre dieser Texten ein Weg zu einer systematischen Ausarbeitung von Huizingas Thesen.

Zunächst sind es einige Punkte, bei denen sich die wichtigsten Übereinstimmungen und Unstimmigkeiten unter die Gesprächspartnern feststellen lassen. Erstens, die Bedeutung und die Rolle des Spiels für das menschliche Leben und die Kultur, die bis Huizinga verkannt wurden. Dieser Sachverhalt wird von allen Autoren implizit oder explizit anerkannt. Die Kulturwissenschaft muss darüber Rechenschaft ablegen, dass sich zunächst nur Psychologie und Pädagogik dem Phänomen Spiel angenähert haben. Das Hauptproblem besteht hier darin, eine eindeutige Bestimmung des Spiels zu finden, angesichts der semantischen Breite des Spielerischen in den verschiedenen Weltsprachen (24). Die Wortfelder in den verschiedenen Sprachen umfassen auch äußerst verschiedene Phänomene. Aufgrund dieser Plastizität schlägt Huizinga eine möglichst weite Definition der Erscheinung Spiel vor, nicht ohne uns zugleich davor zu warnen, dass er nicht die Absicht habe, eine Spieltheorie aufzustellen. Gerade diese Vagheit in der Definition findet Caillois problematisch, denn am Ende habe jede geregelte, konventionelle, willkürliche Form darin Platz. "Seine Formel bezieht sogar die Kriegskunst, die Metrik und die Gerichtsverfahren mit ein« (71). Dagegen schlägt Caillois vor, dass Spiele nach Geisteshaltungen, Geschicklichkeits-, Kombinations- und Kraftspielen in verschiedene Klassen unterteilt werden könnten (61). Wie schon erwähnt, unternimmt Caillois diese Einteilung in Spielgruppen in seinem späten Werk Die Spiele und die Menschen: Maske und Rausch aus dem Jahre 1967. 
Zweitens wird der Ort des Spiels in der menschlichen Kultur strittig, indem Huizinga das Risiko eingeht, Spiel und Kultur gleichzusetzen. Bataille spitzt diese These noch zu: Für Huizinga ist das Spiel nicht bloß ein Kulturfaktor, die Kultur selber ist Spiel (88). Voegelin und Caillois erheben einen fundamentalen Einwand gegen Huizingas Projekt, der sich in dem Vorwurf zusammenfassen ließe, dass durch diese Gleichsetzung das Sakralste der Zivilisation verloren gehen könnte. Dass viele Kulte und Rituale ihren Ursprung im Spiel haben, ist wohl bekannt. Kann man dabei aber von einer ganz problemlosen und linearen Entwicklung ausgehen? So fragt sich Bataille: "Aber musste man nicht beim Übergang von den religiösen Formen (in denen man den tiefen Ernst vor sich hat) zu den willkürlichen, von der unrettbaren Angst losgelösten Formen gerade die Grenze des Spiels unterstreichen?« (79). Nach der Meinung Vogelins lässt sich das Moment der Transzendenz, das alles Spielen auf der Erde beinhaltet, nicht mit dem hegemonialen Naturalismus in den Wissenschaften zusammenbringen (53). Caillois geht mit Huizinga zwar konform in dem Punkt, dass das Spiel und das Heilige sich vom Alltagsleben abgrenzen lassen. Aber Caillois geht davon aus, dass das Spiel und das Heilige sich nicht symmetrisch gegen das Übliche und das Profane verhalten. Sie erfüllen ganz unterschiedliche Funktionen: das Spiel fürchtet das Leben, weil das praktische Leben das Spiel zerstört; das profane Leben fürchtet das Heilige, weil das Leben von der souveränen Macht des Heiligen abhängig ist. Von da aus lässt sich Caillois Überlegungen nach eine Hierarchie Heiliges-Profanes-Spielerisches etablieren. Huizingas letzte Überlegungen in seiner Rektoratsrede lauten: „Der Wissenschaftler ist sich, wie der Künstler, eines Spielelementes in seiner Tätigkeit wohl bewusst. Die reine Wissenschaft, ohne Absicht auf Nutzaufwendung, braucht sich ihres Spielcharakters nicht zu schämen. Sie hat damit Teil am Höchsten, was dem Menschen gegeben ist. Auf Gott bezogen wird alles Menschliche Spiel« (45). Leider blieb diese Anmerkung von den anderen unerhört.

Drittens sind alle Autoren aufgrund der Notwendigkeit, einen Opponenten des Spiels zu finden, dazu gedrungen, das Spielerische innerhalb seiner Grenzen zu halten und dadurch Huizingas geschichtsphilosophische Thesen zu relativieren. Eine wirkungsmächtige Tradition stellt Spiel und Ernst einander gegenüber. Für Huizinga war der Unterschied Spiel-Ernst eher fließend. Vielleicht kann man im Anschluss an Huizinga Ernst und Spiel als zwei Pole eines Kontinuums betrachten. Das Spielerische wird dann dadurch bestimmt, dass die Handlung ihren Zweck in sich selbst hat, während das Ernste heteronom bleibt. Das moderne Spiel der Politik, die Anzeichen einer nationalen Selbstverherrlichung, die Europa abstumpften, diese waren nach Huizinga die Zerstörer der Kultur und des Spiels. Caillois hingegen erblickt die Gefahr des Spiels in anderem. Krieg ist nicht das 
Ende des Spiels: Er stellt lediglich eine andere Form des Spiels dar. Es ist der Falschspieler, der das Spiel gefährdet, der dem Spiel oder der Kultur Ablehnung oder Verachtung entgegenbringt (73). War diese Behauptung gegen Huizingas kulturellen Pessimismus gerichtet? Trotzdem muss hier nochmals daran erinnert werden, dass Caillois der Verführung durch eine zeitgemäße Philosophie des Spiels nicht ganz widerstehen konnte. In seinem Werk Die Spiele und die Menschen: Maske und Rausch sind Mimikry und Rausch die übermodernen Formen des Spiels. Bataille wiederum sieht die Grenze des Spiels in den archaischen kulturellen Verboten gezogen. Diese hätten nichts mit dem Spiel zu tun: Tabus ziehen die Grenzen des Spiels innerhalb der Kultur, d.h. die Grenzen der Unordnung. Die Kultur wie die menschliche Geschichte ist nach Bataille dialektisch auszulegen. Wir befinden uns gegenüber zwei entgegengesetzten dialektischen Modellen: Agon nach Huizinga; Spiel und Arbeit als mögliche Schöpfer der Kultur bei Bataille. Nach Bataille hat Huizinga die Widersprüche nicht angemessen begriffen. Von da aus betrachtet, seien Huizingas Tränen vergeblich und seine Kritik an dem modernen Welt der Arbeit sei schlicht überflüssig. Die Kritik »der negierenden Welt der Arbeit taugt allein dann, wenn sie zunächst einmal einräumt, dass die Welt, die jene des Spiels verneinte, sie nicht irrtümlich verneinte, weil es ihr Wesen war, sondern sie deswegen verneinte, weil es ihr Wesen war, sie zu verneinen " (95). Auch wenn Bataille allzu oft die Leser und Leserinnen darauf aufmerksam macht, dass Huizinga Historiker war (was ihn womöglich als Nicht-Philosophen abstempeln soll) entzieht er sich der geschichtsphilosophischen These jedoch nicht gänzlich. Für Bataille sind Spiel und Souveränität untrennbar mit einander verbunden (102). Souveränität gehöre zu einer anderen Welt; einer Welt, in der souverän zu sein hieße, das eigene Leben aufs Spiel setzen zu können. Unsere Welt habe den Primat der Glorie durch den Primat des Nützlichen ersetzt. Dialektisch betrachtet, wird das Spiel nur wieder einen Platz finden können, wenn die Möglichkeiten der Arbeit bis zu ihrer äußersten Grenze entwickelt seien (111). Heute ist überall von einer Gamification der Arbeitswelt die Rede. Es scheint so, als wären wir schon so weit.

\section{II.}

Die Varianten und Funktionen, die der Spielbegriff im Kontext verschiedener Theorien angenommen hat, lässt sich am besten durch die Begriffsgeschichte rekonstruieren. Auf diesem Weg lassen sich neue Nuancen und sogar unerwartete Affinitäten zwischen Philosophien und Ansätzen entdecken, die sonst nicht anschaulich geworden wären. Der Spielbegriff gewinnt so eine verdiente Zentralität zurück und schafft den Rahmen für traditionelle und innovative Fragestellungen. Der von Michel Henri Ko- 
walewicz herausgegebene Band Spiel. Facetten seiner Ideengeschichte gewährt einen guten Überblick über die Geschichte eines Begriffs, die nicht nur für Historiker immer interessanter wird, sondern auch für Geistes- und Sozialwissenschaftler an Relevanz gewinnt, sofern sie die erkenntnistheoretischen Voraussetzungen ihrer Wissenstätigkeit bewusster herauszuarbeiten gestattet.

Der Spielbegriff scheint einen nicht allzu alten Werdegang in der Deutschen Philosophie absolviert zu haben: Dieser Begriff ist wortwörtlich ein moderner Begriff. Erst nach Kants und Schillers Einführung des Begriffes in die Ästhetik lassen sich Wege für das "freie Spiel der Kräfte» in sehr unterschiedlichen Bereichen wie Wirtschaft, Politik und Moral und ihren korrespondierenden Disziplinen eröffnen. Die Aufnahme dieser partikulären Formel, die das Spiel zwischen individueller Freiheit und sozialer Ordnung einzupassen versucht, findet vor diesem Hintergrund, wie Karl Acham demonstriert, eine sehr breite philosophische Auseinandersetzung über die Grenzen des Spiels. Die Grenzen des Spiels der Freiheit zeigen die Konturen der modernen Welt. Freiheit heisst nicht unbedingt Beliebigkeit oder Nicht-Ernst nach Angelika Hoffman-Maxis. Wir können Schiller hier als Schwelle zwischen der traditionellen Ästhetik als Philosophie des Schönen und der modernen Ästhetik als Philosophie der Kunst betrachten. Die Weiterentwicklung in der Postmoderne, die das Spiel als Schöpfer der Realität vorstellt, mündet in einer Theorie der Fiktion. Fiktion bedeutet nicht Unecht-sein, sondern eher die Schaffung eines freien Umgangs mit der Wirklichkeit ${ }^{22}$. Derart werden auch die Geltungsansprüche des Wirklichen vorübergehend suspendiert und das Spiel wird zum Ernsthaften überhaupt. Spielerisch hat selbst Paul Valéry das eigene Schreiben konzipiert. Seine Literaturtheorie rückt nahe an eine Theorie der Perspektivität heran, deren Inspiration in Leibniz und der mathematischen Spieltheorie liegt. Die Kombination der Augenblicke ist nichts anderes als ein regelhaftes Herstellen des Werkes, was treffend in Valerys Worten zusammengefasst ist: »Ein Gedicht muß ein Fest des Intellekts sein «"23. Es stellt sich an diesem Punkt die Frage, wie es dazu kommen konnte, dass der Ausdruck Spiel synonym für individuelle Freiheit und Kreativität verwendet wird, wenn wir diese Bedeutung mit seiner antiken ontologischen und kosmologischen Prägung konfrontieren. Der Text von Harald Schwaetzer über Nikolaus von Kues bietet ein Bild des Übergangs zwischen diesen beiden Spielbegriffen. Bei Nikolaus von Kues sind vier verschiedene Spielbegriffe zu finden, die Mystik und philosophische Anthropologie mit einander verknüpfen. Spielend ist der menschliche Bezug zur Welt (ludo globi, ludus

\footnotetext{
${ }^{22}$ In diesem Band: Hoffman-Maxis, S. 174-175.

${ }^{23}$ In diesem Band: Lorenz, S. 101.
} 
sapientiae), zu Gott und zum eigenen Schicksal. Es scheint so, als ob der Werdegang des Spiels als Freiheit sich im Horizont der Moderne konstituierte, unterdessen andere Spielbedeutungen ihre Aussagekraft verloren.

Der Begriff tritt erst sehr spät in deutschen philosophischen Wörterbüchern auf, wie Gunter Scholtz in seinem Beitrag reflektiert. Während der Begriff gar nicht oder sehr kurz in den ersten philosophischen Enzyklopädien des 17. und 18 Jahrhunderts erwähnt wird, werden bereits circa 40 Autorennamen in Rudolf Eislers Wörterbuch von 1910 zitiert (239). Was ist den Theoretikern und Philosophen in der Zwischenzeit aufgefallen, so dass die Situation sich derart verändern konnte? Hängt dies mit der Entwicklung der Moderne und der Postmoderne zusammen? Aus Scholtz' Erörterung des Begriffes im Umfeld der Theologie, der Ästhetik, der Anthropologie, der Kulturtheorie und der Entscheidungstheorie ließe sich provisorisch erschließen, dass die Stellung und der Gebrauch des Begriffes sowie die Assoziationen, die der Begriff hervorrufen mag, sehr eng mit dem geschichtlichen und theoretischen Kontext verwoben sind. Sehr interessant ist hierbei auch die Rückgewinnung der theologischen Diskussion über Spiel und Sittlichkeit von Aristoteles bis zum Protestantismus. Dass das Spiel von der Bühne immer wieder verschwindet und immer wieder neue Formen annimmt, macht diese besondere Begriffsgeschichte zu einer theoretisch äußerst anregenden Angelegenheit. Denn diese bedarf nicht nur einer philosophischen Systematisierung der Schwankungen des Begriffes, sondern und vor allem einer interdisziplinäre Annäherung in Anbetracht seiner Vielseitigkeit. Roger Caillois hat dies bereits erkannt in seiner Erforschung des Spiels als Totalphänomen. Er hat sich als Ziel eine Wissenschaft vom Menschen gesetzt, "die ihr Subjekt auch transversal, diagonal und interdisziplinär untersuchen sollte ${ }^{24}$. In Kowalewiczs Darstellung von Caillois Klassifizierung der menschlichen Spiele sind auch die Ursprünge dieser Interdisziplinarität innerhalb der Humanwissenschaften wirksam. Ähnliches kommt bei Simmels Problematisierung des Spiels in seiner Geschichtsphilosophie und seiner Philosophie des Geldes zum Tragen. Annika Schlitte verbindet die Gestaltung einer formalen Soziologe bei Simmel mit seinem Versuch, das Spiel als Erklärungsmuster für andere Phänomene zu gebrauchen. Dadurch musste Simmel nicht nur sein Verständnis von Spiel, Symbolik und Gesellschaft verfeinern, sondern sich im selben Zuge auch bewusster werden über die epistemologischen Grundlagen der Sozialwissenschaft. Dabei ist es vor allem bemerkenswert, wie Simmel vom Spiel als einem individuellen Tun in Probleme der Geschichtsphilosophie ausgeht, bis er es in seinen letzten Werken als ein primär soziales

\footnotetext{
${ }^{24}$ In diesem Band: Kowalewicz, S. 128.
} 
Phänomen betrachtet, um seine Analyse der Geselligkeit als Spielform der Gesellschaft begründen zu können.

Zwei wichtige Linien für die Pfade des Begriffes im 20. Jahrhundert sind besonders präsent in diesem Band: Die phänomenologische Bewegung und die sprachpragmatische Wende, die auf den späten Wittgenstein folgt. Die Beiträge von Annette Sell, Martina Roesner und Pawel Dybel untersuchen sowohl die geschichtsphilosophischen Quellen des phänomenologischen Spielbegriffs als auch seine systematische Funktion innerhalb des philosophischen Denkens Husserls, Heideggers und Gadamers. Die Phänomenologie des Spiels, die sich am besten in der Reihe HusserlHeidegger-Fink-Gadamer verstehen lässt, reanimiert die kosmologische, ontologische, ästhetische und anthropologische Bedeutung des Spiels von Heraklit bis Nietzsche. Martina Roesner, die schon ein hervorragendes Werk über den Spielbegriff bei Heidegger verfasst hat ${ }^{25}$, präsentiert überzeugende Argumente dafür, Husserl als ludi magister der phänomenologischen Tradition wiederzuentdecken. Auf diese Weise lässt sich auch die wesenhafte Dynamik von Sein, Schein und Dasein begreifen. Denn das menschliche Dasein ist das einzige Wesen, dessen Sein dem Rätsel des kosmischen Spiels zugewandt ist. Fink hat dies in einer wunderschönen Formel zum Ausdruck gebracht: "Der Mensch spielt, wo er sein Dasein feiert. ${ }^{26}$ Wittgenstein hingegen wurde stets eine analytische und streng theoretische Auffassung des Spiels als regelhaftes und begrenztes Verfahren zugesprochen. Wie Michael Anacker erläutert, sind die Sprachspiele Lösungen für Sprachverwirrungen, und jedes Problem bedarf einer spezifischen Lösung. Was wir heute von Wittgenstein lernen können, ist, den Handlungsspielraum auszudehnen, wenn wir die Wirklichkeit mit den kleinen Käfigen unseres Verstände einfangen wollen. Dazu gehört auch der Anspruch, jede Region unseres gesellschaftlichen Lebens und seiner jeweiligen Praktiken gelten zu lassen: Wissen, Glauben, Zweifeln und, wie Józef Bremer bemerkt, auch das Beten.

Wittgenstein hat das Konzept der Sprachspiele entworfen, um das komplexe Phänomen der Sprache besser verstehen und sich zugleich besser darüber verständigen zu können. Wir haben es hier mit einem Gebrauch des Spiels zu tun, der nicht in erster Linie metaphorisch ist, sondern etwas Strukturelles mit dem zu erforschenden Phänomen teilt. Einen solchen Gebrauch des Spielbegriffs finden wir vorrangig in den mathematischen und wirtschaftswissenschaftlichen Spieltheorien seit Oskar Morgenstern und Oskar von Neumann. Riccardo Campas höchst interessanter Beitrag

25 Martina Roesner: Metaphysica ludens. Das Spiel als Grundfigur im Denken Martin Heideggers, New York, 2013.

26 Eugen Fink: Grundphänomene des menschlichen Daseins, Freiburg am Breisgau, 1995, S. 414-415. 
über die Spieltheorie, bietet einen Überblick über eine brisante und aktuelle Debatte. Die ökonomische Spieltheorie wuchs als eine Untersuchung von Entscheidungen, die rationale Agenten in Situationen, die konflikthafte oder kooperative Lösungen zeitigen können, treffen. Die strategische Komponente bezeichnet die Game Theory, die sich wiederum von den Kulturwissenschaften und den Game Studies unterscheiden lässt, denn letztere betrachten das Spiel als Totalphänomen. Doch der Ursprung der Gedankenspiele geht nach Campa noch viel weiter zurück: Bereits im Talmud und in Platos Laches sind solche Experimente zu finden (216). Das bekannteste Experiment ist das Gefangenen-Dilemma von Malvin Dresher und Merrill Flood aus dem Jahre 1950. Dieses Spiel wurde konzipiert, um zu bestimmen, wie sich rationale und egoistische Agenten in verschiedenen Kontexten, die Verlust und Gewinn beinhalten, verhalten würden. Die Entwicklung von diesem Experiment bis zu immer raffinierteren Modellen des Ultimatum Game zeigt, wie das Konzept des Homo Economicus zu kurz greift. Synergien der Verhaltensdisziplinen mit den Neurowissenschaften erweitern diese Debatte um weitere Möglichkeiten im Design solcher Experimente und gestatten eine Überprüfung unserer CommonSense Intuitionen. Die Neurowissenschaften sind noch zu jung, um die Reichweite ihrer Resultate ermessen zu können. Zwar ist es sehr informativ zu wissen, dass Oxytocin Großzügigkeit begünstigt und dass Testosteron sie hingegen hindert. Wie diese Information aber zu interpretieren und wie sie anzuwenden ist, bleibt eine ethische Frage. Und dabei kommen ganz andere Erwägungen ins Spiel. Der Homo Economicus ist das hegemoniale Model im Zeitalter des Kapitalismus gewesen: Strategie, Wertsteigerung gehören dazu, wie auch Risiko und Spekulation. Spiele helfen uns zu verstehen, was auf's Spiel gesetzt wird, wie rasch wir gewinnen und verlieren können, oder in wie weit komplexe Systeme übersichtlich und kontrollierbar bleiben. Simone Heinemann zeigt, wie seit Aristoteles Vermögensverwaltung und Spekulation als entgegengesetzte wirtschaftliche Modelle nebeneinander existiert haben, bevor es Bucket Shops oder Warenbörse überhaupt gegeben hat (202-203). Es handelt sich hierbei um zwei Prinzipien, die gemeinsamen Güter und Schäden zu regulieren. Nun ist unser globales Wirtschaftssystem jedoch so komplex geworden, dass es zugleich unkontrollierbar scheint. Daher ist der Ausdruck Kasino-Kapitalismus mehr als eine Metapher dieser zeitgemäßen Komplexität: Er ist das Symbol einer übermodernen Welt. Um von einem richtigen Spiel sprechen zu können, müssten alle Teilnehmer gleichberechtigt dem Spiel beigetreten und an Gewinn und Verlust beteiligt sein. Spiel und Anstand teilen diese Prinzipien. Wie wir wissen, folgt unsere kapitalistische Wirtschaft dieser basalen Spiel- und Anstandsregel jedoch nicht: Eine Minderheit genießt unbegrenzten Gewinn und der mehrheitliche Rest muss da- 
für nicht selten die Rechnung zahlen. Spiel ist Spiel, nur solange sich aus ihm keine wirklichen Konsequenzen für das gewöhnliche Leben ergeben, wie schon Huizinga festgestellt hatte.

\section{III.}

Die übermoderne Gesellschaft erlebt eine beschleunigte Vermehrung der Handlungs- und Spielräume. Ist die Emergenz virtueller Welten eine Chance für schnellere und effizientere Verbindungen oder eher ein vorteilhaftes Szenario für den alten sozialen Maskenball? Sind wir durch soziale Medien offener gegenüber Differenzen geworden oder gar verschlossener als zuvor? Der technische Fortschritt hat Errungenschaften aber auch Herausforderungen mit sich gebracht, die entscheidend für die Erziehung unserer Kinder sein werden. Einerseits vernehmen wir allerorten etwas von Sinnverlust, Nihilismus, Kontingenz, einer Herauslösung aus unserer Geschichte und unseren Lebensbezüge etc. Andererseits fühlen wir uns mit dem ständigen Druck belastet, produktiv und kreativ sein zu müssen in dieser turbulenten Welt. Nicht nur müssen unsere Bekleidungen, Gesten, Haltungen, Gedanken, Meinungen, Ideen, Initiativen und Handlungen originell sein: Unsere Biographie, ja unser Selbst soll als ein Kunstwerk verstanden und geschaffen werden. In einer Gesellschaft, die gern mit Neuigkeiten kokettiert und allzu rasch vergisst, sollen wir unvergesslich und uns selber treu bleiben. In einer Gesellschaft, in der alles seinen Preis hat, sollen wir uns unbezahlbar wähnen. Es ist kein Wunder, dass man die Flucht vor der Realität im Spiel sucht und im Spiel von sich selber Erholung findet.

Dieser Kontext fördert kritische Überlegungen darüber, was das Spiel in der jetzigen Gesellschaften bedeutet und welchen Platz und welche Funktion es einnimmt. Wird alles nur noch verspielt(er)? Die Beiträge in dem von Alfred Schäfer und Christiane Thompson herausgegebenen Band Spiel helfen dieses katastrophale Bild zu relativieren und sich auf weitere Potenziale des Spiels zu besinnen. Diese Potenziale können erst voll ausgeschöpft werden, wenn wir dagegen eine allzu funktionalistische und instrumentalistische Auffassung des Spieles als Teil unserer Arbeitskultur entlarven. Diese Auffassung reduziert das Spielen in der Kindheit auf eine Vorbereitung auf das spätere Leben und die Spiele der Erwachsenen wiederum auf eine Kompensation und bloße Erholung von seriöseren Verpflichtungen. Die Beiträge des Bandes hingegen stehen in der Reihe einer alternativen Tradition, die ebenfalls ihren Ursprung in der Moderne hat: Die ästhetische Tradition Schillers und Nietzsches, die dem Spiel einen Eigenwert zuspricht (10). Sie unterscheiden in dieser ästhetischen Dimension drei Ebenen der Reflexion, deren Implikationen äußerst interessant für das Verhältnis von Spiel, Wirklichkeit und Gesellschaft sind: das Ima- 
ginäre, das Performative und das Subversive im Spiel. Überzeugend ist die Aussage bereits in Schäffers und Thompsons Einleitung: "Spiele reflektieren die Kontingenz, die Grundlegungsproblematik sozialer Ordnungen und korrespondierender Selbstverständnisse« (16).

Das Imaginäre im Spiel transzendiert den Bereich des Realen, indem es eine fiktive Welt erschafft. Fiktion heisst hier nicht bloße Illusion, sondern reflexiver und kritischer Umgang mit dem Realen. Alle Autoren des Bandes gehen letztlich davon aus, dass das Spiel einen Rahmen schafft, durch den übliche lebensweltliche Zwänge in ihrer Geltung suspendiert werden. Die Suspendierung des Üblichen und die Projektion des Imaginären kann sich dabei als Abbild oder auch als Zerrbild des Realen erweisen. Eine fiktive Welt hängt nicht in der Luft: Sie bezieht sich strukturell auf die soziale Welt, in der sie wächst. Anhand der Beispiele von Edvard Munch und Andy Warhol strengt Carl Peter Buschkühle Überlegungen zum spielerischen Experimentieren mit Techniken, Formen und Inhalten der Kunst an, durch die das künstlerische Denken und Schaffen einen Übergang von der Potenzialität zur Aktualität herstellt (80-81). Kunstwerke sind dann mehr als ihre Zeit in einem Abbild erfasst: Sie enthalten überdies Sinnpotenziale, die in ihr noch nicht realisiert wurden. Dieser Übergang ist nur denkbar, wenn die Sinnpotenziale in der jeweiligen Zeit nicht ein für alle mal eingeschlossen bleiben. Das Ausklammern von Gewissheiten ermöglicht die Eröffnung von Gestaltungsmöglichkeiten, weil das Gewebe unserer Kultur porös und vielschichtig ist. Aufgrund dieser strukturellen Erschließung des Realen ist laut Steffen Wittig das Spiel heute wie zuvor der Modus jeden Umgangs mit Kultur: "Da Kultur in Form eines heterogenen und kontingenten Möglichkeitsraum existiert, muss sich das Subjekt spielend $\mathrm{zu}$ ihr verhalten. Eine endgültige Sinnfixierung scheint unmöglich und dennoch verschreibt sich das Subjekt Möglichkeiten des Sinns: Das Subjekt wird zum Spieler, welcher jedoch nicht nur mit der Differenz in der Ordnung des Sinns umgehen muss, sondern auch mit der Differenz im Selbst« (180).

Cristoph Wulf identifiziert unter den vielen Funktionen des Rituellen auch die Bearbeitung und den Umgang mit der Differenz (103). Bereits Huizinga in Homo Ludens hat Spiele und Rituale als Grundelemente der Kultur erforscht; die Hierarchie der beiden bleibt jedoch unklar. Wulfs Auffassung von Ritualen ist dagegen weit genug, um die ludische Komponente im Rituellen anzuerkennen, ohne aber Spiele und Rituale auf einander zu reduzieren. Rituale werden als Formen der Performativität verstanden, die Körperlichkeit involvieren und daher mehr soziales Gewicht haben. Als Formen der Performativität sind Rituale kommunikative kulturelle Ausführungen, die eine ästhetische Dimension besitzen und Gemeinschaften durch Integration und Exklusion bilden. Nach Wulf be- 
steht zwischen der internen Ordnung von Spielen und der Ordnung der Gesellschaft, in der sie inszeniert und aufgeführt werden, ein mimetisches Verhältnis (99). Spiele und Rituale haben als performative Handlungen mimetische Prozesse gemein. Diese Mimesis ist aber semantisch nicht eindeutig, sie enthält einen Bedeutungsüberschuss, der über den institutionalisierten sozialen Rahmen hinausgeht. Daher sind nach Wulf Rituale nicht allein Handlungen, die die gesellschaftliche Organisation bewahren, sondern innerhalb ihrer können sie auch Innovationen begünstigen. Es wäre aufschlussreich, diesen Gedanke weiterzuverfolgen und die rituellen Bedingungen für soziale Innovationen genauer unter die Lupe zu nehmen.

Die Entstehung des Social Gaming bietet hierzu einen hervorragenden Gegenstand. Gelingt es dem medialen Umfeld, eine radikal innovative Spielform neben den traditionellen analogen Spielformen zu etablieren? Jens Holze und Dan Verständig gehen dieser Frage nach mit der Überprüfung und Übertragung vor-digitaler Spielmerkmale auf die digitale Umgebung. Durch die Einführung technischer Innovationen können Ausdehnung, Intensivierung und Transformation in mehreren Bereichen der sozialen Arena festgestellt werden. Dabei sind die meisten Veränderungen auf strukturelle Gegebenheiten des digitalen Spiels zurückzuführen. Trotzdem scheint es den Autoren noch zu früh, die Ludifizierung des Sozialen umfassend zu bewerten. Das Spielerische im Zeitalter des Internets hat aber eine positive Note: Internet stellt einen deliberativen Rahmen bereit, der die Qualität des Spiels und des Spielerischen verändert (153). Die Qualität des Spielerischen wird als komplexe Struktur von Technik, Kultur und Identität gefasst (140). Als Mead ${ }^{27}$ und Goffmann ${ }^{28}$ den Nexus zwischen Identitätsbildung, Rollenzuschreibung und Selbstdarstellung hervorhoben, war die Anzahl der sozialen Bühnen noch um einiges begrenzter. Jetzt haben Individuen mehrere Plattformen, um sich der Öffentlichkeit zu zeigen, aber auch um sich in der Anonymität zu verstecken. Haben wir es im Internet mit der Gefahr einer Auslöschung und Dezentrierung des Selbst zu tun? Begünstigt die Gamification der Lebenswelt die Herstellung von Pseudo-Identitäten und verflüssigten Verbindlichkeiten? Gabriele Weiß thematisiert die problematische Grenzziehung zwischen Spiel und lebensweltlicher Praxis ausgehend von der Stellung der Subjektivität im Spiel. Echte Spieler lassen sich auf das Spiel ein und bewegen sich im Rahmen der vereinbarten Spielregeln. Anders verhalten sich Falschspieler oder Spielverderber. Ersterer tut so, als würde er spielen, während

27 George Herbert Mead: Mind, Self, and Society from the Standpoint of a Social Behaviorist, Chicago, 1967.

28 Erving Goffman: Wir spielen alle Theater: Die Selbstdarstellung im Alltag, München, 2003. 
er das Spiel als Instrument seiner Dominanz und Souveränität missbraucht. Letzterer versucht hingegen, den Rahmen des Spiels zu sprengen und dadurch das Spiel in Frage zu stellen. In den Worten von Weiß: „Falschspieler und Spielverderber haben die Distanz gemeinsam, sie sind nicht im Spiel versunken und tun nur so, als seien sie am Spiel interessiert. Der Spielverderber zeigt seine Haltung dem Spiel gegenüber, der Falschspieler verbirgt seine distanzierte Gelassenheit, so dass die Illusion für andere bleibt« (46). Wenn wir die Analogie von Leben und Spiel erweitern, multipliziert und intensiviert die Einführung der Medialität in Prozesse von Selbstdarstellung und Selbstinszenierung die Chancen für das falsche Spielen mit anderen und mit sich selbst. Es sind aber auch Chancen vorhanden, kritisch, selektiv und experimentell mit Möglichkeiten des Seins und des Nichtseins zu spielen. Im Leben wie im Spiel erlaubt uns die Beweglichkeit des Seins nicht permanent in derselben Stellung zu verharren: eher sind wir einmal distanziert, einmal involviert und einmal gleichgültig gegenüber dem, was es uns zuspielt.

\section{IV.}

Der Übergang von einem antiken zu einem modernen Spielbegriff erfolgt durch eine Schwerpunktverlagerung in der Konstellation von Sein, Spiel und Subjekt. Der antike Spielbegriff bezieht sich auf ein kosmisches Spiel, das als Weltsymbol fungiert ${ }^{29}$ und demgegenüber die Endlichkeit des menschlichen Wesen betont. Die moderne Philosophie stellt das menschliche Subjektivität ins Zentrum einer Welt, die es zu erkennen, zu bearbeiten und zu meistern vermag. Kant hat diese Rotationsbewegung »kopernikanische Wende« genannt. Das Spiel wird dann zum Symbol der subjektiven Freiheit mit Blick auf die Sinnbildung. Der von Regine Strätling herausgegebene Band Spielformen des Selbst hat als Ausgangspunkt die Korrelation von Subjektreflexion und Spielreflexion. Der Band zielt auf eine künftige Genealogie der Spielforschung und der abendländischen Subjektivität, wobei durch den modernen Rekurs auf den Spielbegriff ein Zusammenhang mit den Identitätsstiftungsproblemen des Subjekts hergestellt würde. Sie ist noch zu schreiben (10). Die Postmoderne hat diese Korrelation verschärft und vertieft: Hierfür finden sich im Band äußerst interessante Beispiele aus der Philosophie ${ }^{30}$, aus der Psychologie ${ }^{31}$, aus der

\footnotetext{
29 Eugen Fink: Spiel als Weltsymbol, Freiburg am Breisgau, 2010.

30 Siehe in diesem Band: Stefan Deines, Natascha Adamowsky, Sandra Markewitz, Somogy Varga, Kyung-Ho Cha

31 Siehe in diesem Band: Julia Christ, Somogy Varga.
} 
Kunst $^{32}$, aus der Literatur ${ }^{33}$, aus der Kulturwissenschaft ${ }^{34}$, aus der Medienwissenschaft ${ }^{35}$ und aus der empirischen Sozialwissenschaft ${ }^{36}$. Ein wichtiges gemeinsames Ziel dieser Beiträge ist laut der Herausgeberin: »den Begriff des Spiels zur Bestimmung der Medialität im Zusammenhang von Subjektivierungsprozessen operativ einzusetzen sowie ausgehend von konkreten Spielen bzw. als Spiel zu begreifenden Praktiken deren Rolle in solchen Prozessen auch medienphilosophisch zu reflektieren" (17).

Eine der Hauptschwierigkeiten für diese gemeinsame Genealogie der modernen Subjektivität und des modernen Spielbegriffs besteht jedoch darin, dass trotz eines Faibles einiger prominenter Philosophen (wie Nietzsche, Heidegger, Mead, Gadamer, Derrida und Brandom) für das Spiel sich die Spielphilosophie oder die Ludologie neben den anderen philosophischen Disziplinen nicht behaupten konnte. Stattdessen wurden Spiele in der Philosophie eher als Modelle oder Metaphern für andere Phänomenen herangezogen, ohne den Spielbegriff selbst zu hinterfragen. Der Mangel an einem einheitlichen Spielbegriff mag eine Erklärung dafür bieten, warum es bis heute keine philosophische Ludologie gegeben hat. Aber diese Erklärung ist nicht ganz überzeugend: Denn wir haben auch keinen einheitlichen Begriff des Guten und Bösen, des Schönen oder der Erkenntnis und trotzdem besitzen wir eine philosophische Ethik, eine philosophische Ästhetik, Erkenntnistheorie und Wissenschaftsphilosophie. Vielleicht liegt der Unterschied darin, dass es bei diesen anderen Begriffen eine schon klar definierte Debattenebene gibt, auf der der theoretische und normative Konsens bzw. Dissens sich wechselseitig stützen können. Die Offenheit des Spielens schließt diese Eingrenzung eines geschlossenen Bereich gerade aus. Die Spielforschung ist durch ihre notwendige Multidimensionalität bedingt. Daher scheint mir Stefan Deines Vorschlag, durch die Überprüfung verschiedener Funktionen des Spielbegriffs das Offene und Facettenreiche des Spielbegriffs aufrechtzuerhalten, in der Philosophie und in der Konsequenz vielversprechend $\mathrm{zu} \operatorname{sein}^{37}$. Deines

32 Siehe in diesem Band: Céline Kaiser, Christian Moser,

33 Siehe in diesem Band: Renate Strätling.

34 Siehe in diesem Band: Mario Bührmann.

35 Siehe in diesem Band: Hans-Christian von Hermann, Michael Liebe, Markus RAUTZENBERG.

${ }^{36}$ Siehe in diesem Band: Bernd Ternes, Volker Woltersdorff.

37 Andere interessante Versuche in dieser Richtung wären, unter anderen: die schon eingeführte »rhetorische Lösung« von Brian Sutton-Smith; der pluralistischen Spielbegriff von Randolph Frenzell (Randolph Frenzell: A pluralist concept of play, in: The philosophy of play, hg. Von E. Ryall, W. Russell, M. Maclean, London, 2013, S. 11-31); oder Chris Batemans ästhetische Topographie von Games (Chris Bateman: A disavowal of games, in: Philosophical Perspectives of Play, hg. Von E. Ryall, W. Russell, M. Maclean, London, 2015, S. 71-83). 
verfolgt zwei Strategien, die sich als fruchtbar erweisen könnten. Erstens skizziert er die Grundzüge einer Typologie des Spiels, die drei verschiedene Dimensionen des Spiels in Erwägung zieht: Spielformen (im Anschluss an Caillois, Wettkampfspiel, Glücksspiel, Darstellungsspiel, freies Spiel), Akteure (die Subjekte im Spiel, die nicht immer von vorne herein identifizierbar sind), Sphäre (die Grenzen des Spiels können wohl definiert oder weitschweifig sein, was wiederum eine vertikale-strenge Differenzierung voraussetzt oder eine horizontale-ausgedehnte Breite des Spiels in der Kultur erlaubt). Zweitens unternimmt Deines eine Klassifizierung der Spielphilosophien nach ihren Verwendungsweisen des Spielbegriffs in der Philosophie der Moderne und der Postmoderne, die verschiedene Aspekte der Spieldimensionen betonen oder bevorzugen. Aus dieser Überschneidung von Spieldimensionen und Spielbegriffen resultieren vier unterschiedliche Gebräuche des Spiels und ihrer dementsprechenden theoretischen Funktionen innerhalb der philosophischen Ansätze: (I) Spielphilosophie im engeren Sinne bei Mead oder Huizinga - der Mensch als kreative und kulturschaffende Kreatur. (II) Die ästhetische Tradition im Anschluss an Kant - ein freies Subjekt, das passiv und rezeptiv im Vollzug der Erfahrung erscheint (III) Analytische und postanalytische Sprachphilosophie Regelfolgen und richtiger Verlauf des Spiels. (IV) Hermeneutik und Dekonstruktion - Diagnose der Kontingenz von Weltverständnissen und Weltverhältnissen. Die Gebräuche des Spielbegriffs implizieren gleichwohl alternative Selbstverständnisse und Handlungsmöglichkeiten. Keine dieser Traditionen entstand von sich aus, gleichsam isoliert. So wäre es für die Spielforschung erforderlich, die Überschneidungen und Distanzierungen dieser Denktraditionen weiterzuverfolgen.

Dass der Spielbegriff derart geeignet scheint als Modell oder Metapher für komplexe Phänomenen, verdankt er seiner intrinsischen Plastizität und seinem Vermittlungspotenzial. Metaphern sind selber eine Art Spiel, die zwei Gegenstände in einem Ausdruck wegen ihrer strukturellen Analogien zusammenfügen, ohne dass wir das "als ob«, die konstitutive Differenz zwischen den Polen der Gleichung, vergessen würden. Metaphern sind demnach spielerische Ressourcen unserer Sprache und unserer Einbildungskraft, Gleichheiten und Ungleichheiten zu erkennen. Besonders eindrücklich hierzu ist Adamowskys Beitrag zu der "Kunst des Findens und dem Spiel des Zeigens « in ästhetisch-medialen Prozessen, die »eine Zwitterstellung des Subjekts erkennbar [macht], die mit der Ambivalenz der Dinge, sprich des Gefundenen als Gemachten wie Eigensinnigen, korrespondiert« (75). Strätlings Einführung in die Projekte eines biographischen Schreibens des Selbst in der Französischen Literatur des 20. Jahrhunderts hat es mit einer gesuchten und geförderten Vielschichtigkeit des Selbst zu tun, die aus der Skepsis gegenüber einer Ausdeutbarkeit des Selbst ent- 
springt (183). In der Philosophie und in der Literatur wurde demnach schon viel früher das Terrain sondiert, um die moderne Subjektivität nicht nur als souveräne Selbstbestimmung aufzufassen, sondern, auch um Prozessen der Selbstzentralisierung und Selbstdezentralisierung Beachtung zu schenken. Diese Prozesse haben durch die Figur des Avatars in Computerspielen eine weitere Entwicklung durchlaufen. Michael Liebes Beitrag legt dar, wie der aus dem hinduistischen Mythologie stammende Begriff in seiner ursprünglichen Bedeutung als Inkarnation des Göttlichen eine Erweiterung in der Verwandlung von menschlichem Spieler zum virtuellen Spieler erfahren hat. Der Avatar ist der Vertreter des Spielers im Computerspiel. Vergleichbar der Gottheit Vihsnu bleibt der Spieler gewissermaBen unsterblich, unterdessen sein avat ra sich irdischen Gefahr aussetzt (208). Dabei liefert der Avatar jedoch eine Form der Präsenz für den Spieler im Spiel, die das Medium seiner Erfahrung darstellt. Wie die hinduistischen Gottheiten ist der Computerspieler von den materiellen Bedingungen der Umgebung abhängig. In diesem Fall können Bewegungsfreiheit und Handlungsraum des Avatar-Spielers sehr streng von den technischen Gegebenheiten des Spieles bedingt sein: "Im Computerspiel ist Fiktion eingebettet in ein Set von Regeln, Aktionsmöglichkeiten, Spielzielen und Konflikten" (214). Der Charakter des Avatar-Spielers wird in der Auseinandersetzung mit den medialen Einschränkung des Spiels geprägt. Die Spielregeln werden durch das Computersystem aufrechterhalten, was theoretisch die Kohärenz des Spiels nicht gefährden sollte. Nur Spielweisen wie beispielsweise der Speedrun (wenn ein Spieler von Half Life oder Super Mario World, statt den ludischen Konflikt zu lösen, mit seinem Avatar lediglich so schnell wie möglich rennt) überschreiten das geschlossene Spielsystem und etablieren ein Metaspiel (218). Solche Phänomene werden am besten erklärt, wenn man Computerspiele sowohl als Spiele als auch als ästhetische Gegenstände auffasst, die durch ihre Medialität und Prozessualität definiert werden. Markus Rautzenberg betrachtet beide Aspekte in seiner Analyse der ludischen Medialität und Spielererfahrung in Computerspielen. Nach Rautzenberg ist die ästhetische Gegenständlichkeit des Computerspiels auf der Ebene des Spielgeschehens zu suchen und nicht auf der Bildebene, der Narration oder der dargestellten Gewalt: „Computerspiele werden wie alle Spiele nicht gelesen oder wie ein Film rezipiert, sondern aktiv exploriert, mit ihnen wird praktisch umgegangen, ja sie existieren nicht ohne dieses praktische Handeln des Spielers" (239). So sind Interaktionsmöglichkeiten mit dem Medium und mit anderen Spielern korrelativ zu den Möglichkeiten des Selbst. Allerdings ist dieser Prozess noch im Gange und es ist noch zu früh, die Zukunft des Spielens im Zeitalter der digitalen Spiele zu prognostizieren oder gar Anklagen zu erheben gegen den Grad der Gamification in unserer Gesellschaft. Stattdes- 
sen scheint die Zeit jedoch schon reif dazu, dem Ludic Turn auch in der Philosophie mehr Aufmerksamkeit zu schenken. Abschließend kann man Natascha Adamowsky wohl nur zustimmen: "Zu spielen heißt, Verbindungen herzustellen, spekulative Brücken zu schlagen, Disparates zusammenzuführen. Spiele stellen ein kulturanthropologisches Archiv von Ausdrucksweisen bzw. Begegnungsformen zur Verfügung, die es ermöglichen, etwas zu etwas anderen in Beziehung zu setzen. Sie können glücken oder auch nicht, in jedem Fall hat man es mit einer Dynamik des Lebendigen zu tun« (62).

Núria Sara Miras Boronat Universidad de Barcelona Departament de Filosofia Montalegre 6 08001 Barcelona nsmiras@ub.edu 
